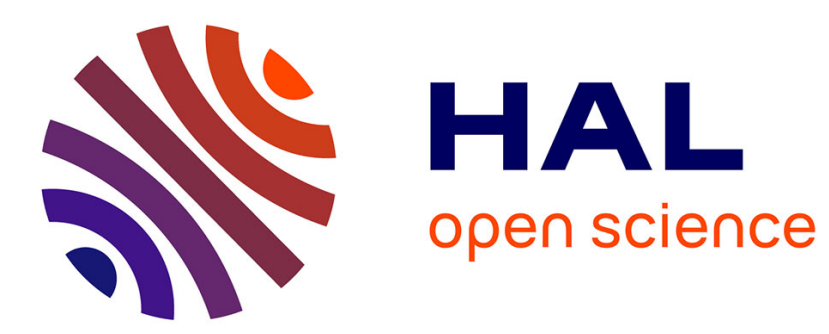

\title{
Computational Algorithm for Some Problems with Variable Geometrical Structure
}

Nikolai Bessonov, Vitaly Volpert

\section{To cite this version:}

Nikolai Bessonov, Vitaly Volpert. Computational Algorithm for Some Problems with Variable Geometrical Structure. Lecture Notes in Computer Science, 2011, 6260/2011, pp.87-102. 10.1007/978-3642-16236-7_6. hal-00653652

\section{HAL Id: hal-00653652 https://hal.science/hal-00653652}

Submitted on 19 Dec 2011

HAL is a multi-disciplinary open access archive for the deposit and dissemination of scientific research documents, whether they are published or not. The documents may come from teaching and research institutions in France or abroad, or from public or private research centers.
L'archive ouverte pluridisciplinaire HAL, est destinée au dépôt et à la diffusion de documents scientifiques de niveau recherche, publiés ou non, émanant des établissements d'enseignement et de recherche français ou étrangers, des laboratoires publics ou privés. 


\title{
Computational Algorithm for Some Problems with Variable Geometrical Structure
}

\author{
N. Bessonov ${ }^{1}$, V. Volpert ${ }^{2}$ \\ ${ }^{1}$ Institute of Mechanical Engineering Problems, 199178 Saint Petersburg, Russia \\ ${ }^{2}$ Institut Camille Jordan, UMR 5208 CNRS, University Lyon 1 \\ 69622 Villeurbanne, France
}

\begin{abstract}
The work is devoted to the computational algorithm for a problem of plant growth. The plant is represented as a system of connected intervals corresponding to branches. We compute the concentration distributions inside the branches. The originality of the problem is that the geometry of the plant is not a priori given. It evolves in time depending on the concentrations of plant hormones found as a solution of the problem. New branches appear in the process of plant growth. The algorithm is adapted to an arbitrary plant structure and an arbitrary number of branches.
\end{abstract}

Key words: plants, branching, variable structure, computational algorithm

\section{Introduction}

Plant modelling attract much attention both from the point of view of understanding of fundamental biological mechanisms and for practical purposes related crop optimization. Various approaches are developed (see [1], [2]). In this paper we describe a numerical algorithm developed in order to model plant growth and structure formation on the basis of realistic biological mechanism suggested in our previous works [3], [4]. Plants are considered here as interconnected one-dimensional intervals. Each interval corresponds to a branch. There are branches of different generations. There exists only one branch of the first generation. It corresponds to the trunk. One its end is fixed and corresponds to the root, another one moves with some speed. If we introduce a space variable $x$ along this interval, then $x=0$ corresponds to its fixed end and $x=L(t)$ to the moving boundary. We do not consider the root system and take it into account only by means of the flux of nutrients passing though the fixed boundary. The moving boundary corresponds to the apical meristem, a narrow layer of cells at the very end of the growing branch. These cells divide and determine the plant growth. The cells along the trunk differentiate and do not divide anymore.

We next introduce the concentration of nutrients $C(x, t)$, which depends on the space 
variable $x$ and on time $t$, and the concentration of growth and mitosis factor $R(t)$, which depends only on time. Nutrient are supplied trough the fixed boundary of the interval and consumed at its moving boundary. Growth and mitosis factor is located in the apex. It is produced there and determines the proliferation rate of the cells, that is the rate of branch growth. Thus, we obtain a free boundary problem where the speed of the boundary is determined by the variable $R(t)$ defined at this boundary and by the variable $C(x, t)$ defined in the whole interval. Precise formulation of the problem will be given in the next section. Biological background of this study is presented in the previous works [3], [4].

Branches of the second generation are also straight intervals with one endpoint inside the first branch (trunk) and another endpoint, which is free. It corresponds to the apex of this new branch and determines its growth. The number of branches of the second generation can be arbitrary, as well as their starting points. These are not fixed parameters of the problem.

In order to describe how branches of the second and of other generations appear, we briefly recall some biological facts. Appearance of new buds is determined by the concentrations of plants hormones auxin and cytokinin. They are produced by the plant itself and redistributed by ascending or descending fluxes. The most recent investigations reveal that auxin efflux carrier PIN1 protein plays the central role in this regulation. The expression of this protein is considered as a key factor for formation of plant organs [5]-[10], [11]-[17]. On the other hand, regulation of the PIN genes is itself under the auxin control (via PLETHORA gene feedback loop [13]). Therefore, we have an auxin induced auxin efflux which can be modelled with or without the intermediate PIN protein. However, auxin by itself cannot initiate cell proliferation. It can happen only in the presence of cytokinin, another plant hormone (actually a group of hormones), the main role of which is the regulation of cell proliferation. Therefore it can be an interplay of these two hormones that causes formation of a new bud.

Thus, if these concentrations take on some given values at some space point, then a new bud appears there. From the algorithmic point of view, buds represent very short branches with associated values of the concentration of mitosis and growth factor. If this value is low, then the bud remains dormant. If it becomes sufficiently high, then the bud gives a new growing branch. Thus, we introduce two new variables $A(x, t)$ and $K(x, t)$ which describe the distributions of auxin and cytokinin. They are defined inside all branches, as well as the concentration of nutrients $C(x, t)$. The space variable $x$ here is proper to each branch. A more precise description of the model is given below.

We summarize the model of plant growth as follows. Plant is represented as a number of connected and growing straight intervals. Their appearance, location and the rate of growth are determined by concentrations of nutrients, hormones and mitosis and growth factors defined either inside the branches or at their boundaries. The plant architecture, that is the number of intervals and their locations are not a priori given. From the mathematical point of view, we consider several free boundary problems whose solutions influence each other. The number of these problems and their relation to each other evolve in time. This is a new type of free boundary problems which we call one-dimensional problems with branching. The 
numerical algorithm should be able to describe any possible plant architecture. The main purpose of this work is to present this algorithm (Section 3). We illustrate its application to the study of branching patterns in Section 4.

\section{Model}

\subsection{Without branching}

We consider in this work one-dimensional model justified if the length (or height) $L$ of the plant is essentially greater than the diameter of its trunk. Hence we consider the interval $0 \leq x \leq L(t)$ with the length depending on time. The left endpoint $x=0$ corresponds to the root. Its role is to provide the flux of nutrients taken into account through the boundary condition. We do not model the root growth here in order to simplify the problem. Therefore the left boundary is fixed. The right endpoint, $x=L(t)$ corresponds to the apex. Its width is much less than that of the plant. We suppose in the model that it is a mathematical point. The value $L(t)$ increases over time. According to the assumption above, the growth rate is determined by the concentration of metabolites at $x=L(t)$, which we denote by $R$. Thus

$$
\frac{d L}{d t}=f(R)
$$

The function $f(R)$ will be specified below.

We recall that the interval $0<x<L(t)$ corresponds to differentiated cells that conduct nutrients from the root to the apex. We suppose that they are in a liquid solution. Denote by $C$ their concentration, which is a function of $x$ and $t$. Its evolution is described by the diffusion-advection equation

$$
\frac{\partial C}{\partial t}+u \frac{\partial C}{\partial x}=d \frac{\partial^{2} C}{\partial x^{2}}
$$

Here $u$ is the velocity of the fluid, and $d$ is the diffusion coefficient. Assuming that the fluid is incompressible and fills the xylem uniformly (the part of the plant tissue conducting nutrients from below to above and located inside the cambium layer), we obtain

$$
u=\frac{d L}{d t}
$$

We complete equation (2.2) by setting the boundary conditions

$$
x=0: C=1, \quad x=L(t): d \frac{\partial C}{\partial x}=-g(R) C .
$$

The second boundary condition shows that the flux of nutrients from the main body of the plant to the meristem is proportional to the concentration $C(L, t)$. This is a conventional 
relation for mass exchange at the boundary, Robin boundary conditions. The factor $g(R)$ shows that this flux can be regulated by proliferating cells. We discuss this assumption as well as the form of the function $g(R)$ below.

We now derive the equation describing the evolution of $R$. At this point we need to return to the model in which the width of the meristem is finite. We denote it by $h$. Then we have

$$
h \frac{d R}{d t}=g(R) C-\sigma R
$$

The first term in the right-hand side of this equation describes production of the GM-factor $R$ in the meristem. The second term corresponds to its consumption.

System of equations (2.1)-(2.4) is a generic one-dimensional model of plant growth based on: a) "continuous medium" assumptions of mass conservation (for $C+R$ ) and of the proportionality of the flux $\partial C / \partial x$ at the boundary to the value of $C$; and b) a "biological" assumption that there is a chemical species $R$, the plant growth and mitosis factor, which is produced in the meristem and which determines the plant growth.

We note that the conservation of mass in the case $\sigma=0$ implies that the term $g(R) C$ enters both the boundary condition and equation (2.4). Therefore, the assumption that the rate of the plant growth factor production depends on its concentration $R$ makes the boundary condition depend on it also. We will see below that properties of the function $g$ can be crucial for plant growth. In particular, if it is constant (the production rate is not auto-catalytic), we will not be able to describe the essential difference in plant sizes.

We now specify the form of the functions $f$ and $g$. We will consider $f$ as a piece-wise constant function equal to 0 if $R$ is less than a critical value $R_{f}$ and equal to some positive constant $f_{0}$ if $R$ is greater than $R_{f}$ (Figure 1a). This means that growth begins if the concentration of the plant growth factor exceeds some critical value.

The production of the growth factor $R$ is assumed to be auto-catalytic. To simplify the model, we consider a piece-wise linear function $g(R)$ (Figure 1b). In some cases we also consider smooth functions $f$ and $g$. These assumptions are consistent with plant morphogenesis. They are discussed in [3], [4] in more detail.

\section{$2.2 \quad$ With branching}

The concentrations of nutrients $C$, and of hormones $A$ and $K$ are described by the diffusion equations with convective terms:

$$
\begin{gathered}
\frac{\partial C}{\partial t}+V \frac{\partial C}{\partial x}=d_{C} \frac{\partial^{2} C}{\partial x^{2}} \\
\frac{\partial K}{\partial t}-V_{K} \frac{\partial K}{\partial x}=d_{K} \frac{\partial^{2} K}{\partial x^{2}}-\mu K
\end{gathered}
$$



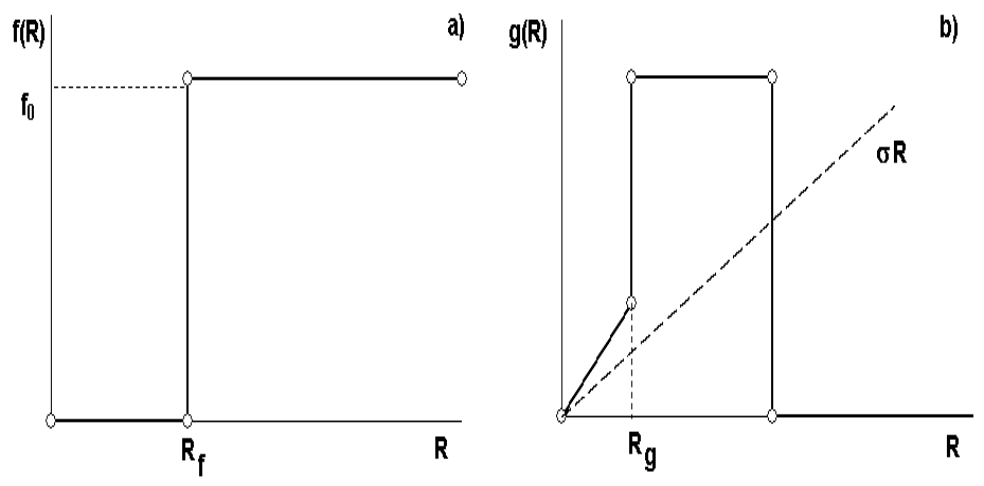

Figure 1: Functions $f$ and $g$.

$$
\frac{\partial A}{\partial t}-V_{A} \frac{\partial A}{\partial x}=d_{A} \frac{\partial^{2} A}{\partial x^{2}}-\mu A .
$$

The convective speed $V_{A}$ in equations (2.6) and (2.7) can be different in comparison with equation (2.5). It corresponds to transport in the phloem in the direction from top (meristem) to bottom (root). The speed $V$ in the first equation is determined as the speed of growth:

$$
\frac{d L}{d t}=V,\left.\quad L\right|_{t=0}=L_{0}, \quad h \frac{d R}{d t}=C g(R)-\sigma R,\left.\quad R\right|_{t=0}=R_{0}, \quad V=f(R)
$$

Here $d_{C}, d_{K}, d_{A}$ and $\mu$ are parameters; the space variable $x$ is defined independently for each branch.

The boundary conditions for $C$ are the same as in the case without branching:

$$
\left.C\right|_{x=0}=C_{0}, \quad d_{C} \frac{\partial C}{\partial x}+\left.C g(R)\right|_{x=L}=0 .
$$

The boundary conditions for $K$ describe its possible production in the root, and its production in the meristem with rate proportional to the rate of growth:

$$
\left.K\right|_{x=0}=K_{0},\left.\quad d_{K} \frac{\partial K}{\partial x}\right|_{x=L}=\varepsilon V .
$$

Finally, the boundary conditions for $A$ are similar, except that the boundary condition at $x=0$ takes into account that this horomone can be transported from the stem to the root:

$$
\frac{\partial A}{\partial x}-\left.\beta A\right|_{x=0}=0,\left.\quad d_{A} \frac{\partial A}{\partial x}\right|_{x=L}=\varepsilon V .
$$

We define next the branching conditions. A new branch appears at $x=x_{0}$ and $t=t_{0}$ if

$$
A\left(x_{0}, t_{0}\right)=A_{b}, \quad K\left(x_{0}, t_{0}\right)=K_{b},
$$


where $A_{b}$ and $K_{b}$ are some given values. Appearance of a new branch means that there is an additional interval connected to the previous one at its point $x_{0}$. The variables $C_{n}, A_{n}, K_{n}$ and $R_{n}$ are described at the new interval by the same equations as above. Here the subscript $n$ determines the number of the branch. We should complete the formulation by the initial value of the concentration $R_{n}=R_{n}\left(t_{0}\right)$. It cannot be found as a solution of the problem but should be considered as a parameter.

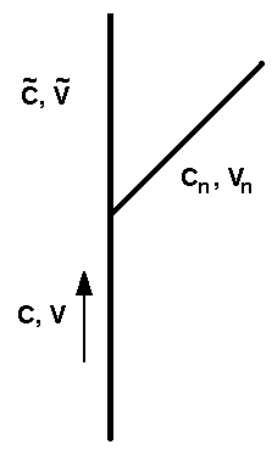

Figure 2: Schematic representation of convective and diffusion fluxes in 1D plant with branching.

There are additional branching conditions for the concentrations

$$
C_{n}(0, t)=C\left(x_{0}, t\right), \quad A_{n}(0, t)=A\left(x_{0}, t\right), \quad K_{n}(0, t)=K\left(x_{0}, t\right),
$$

which means that the concentrations are continuous at branching points. We also need some conditions on the fluxes to provide the conservation of mass. Under the notations shown in Figure 2, we have

$$
S V=\tilde{S} \tilde{V}+S_{n} V_{n}, \quad S \frac{\partial C}{\partial x}=\tilde{S} \frac{\partial \tilde{C}}{\partial x}+S_{n} \frac{\partial C_{n}}{\partial x} .
$$

Here $S, \tilde{S}$, and $S_{n}$ are parameters determined by the cross section area of the corresponding branch. If all branches have the same cross section, then $S=\tilde{S}=S_{n}=1$. If the cross section areas are conserved and narrow branches have the same diameter, then $S=1$, and $\tilde{S}=S_{n}=1 / 2$.

In this work we restrict ourselves to the case where all branches have the same cross section. Otherwise, the diameter of each branch should be considered as a function of time. The diameter would depend on fluxes of metabolites coming through the branch to the root. On the other hand, the fluxes of nutrients going through the branch from the root would depend on its cross section. We obtain a very complex time-dependent problem with many 
feedbacks. This will essentially complicate the understanding of the mechanism of growth and the interpretation of the results.

As is discussed in the previous section, bud formation is accompanied by production of $A$ and $K$. The angle of the new branches with respect to the previous one is given as a parameter. It is not related to light and photosynthesis which are not considered in this model.

\section{Computational algorithm}

In this section we discuss the numerical algorithm for the one-dimensional problem with branching. The model is based on the reaction-diffusion equations, which can be considered in the following form:

$$
\frac{\partial \varphi}{\partial t}+u \frac{\partial \varphi}{\partial x}=d \frac{\partial^{2} \varphi}{\partial x^{2}}-\mu \varphi
$$

where $\varphi$ corresponds to the unknown variable (e.g. $C, K), x$ is the space variable defined on each particular branch. Let us introduce a finite-difference mesh along any branch with the nodal coordinates $x_{i}(i=1, \ldots, I)$ (Figure 3a).

a)

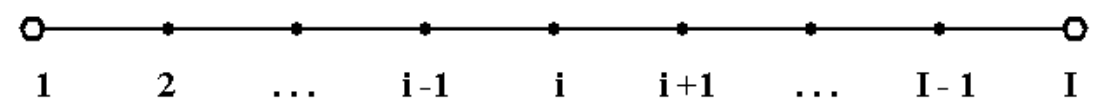

b)

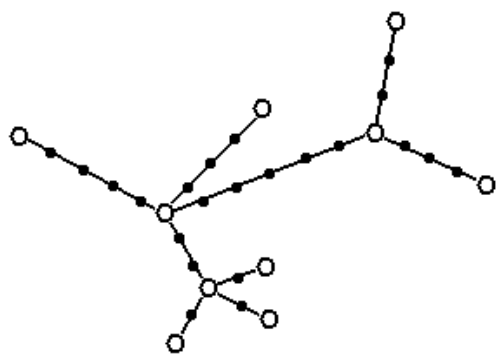

O boundary node

- internal node

Figure 3: Numerical mesh with branching

Let the nodes $x_{i}$ be located uniformly with distance $\delta x$ between them. We will denote the values of $\varphi$ in the nodes by $\varphi_{i}$. Consider an explicit upwind approximation for equation (3.13) first in the interior nodes $(i=2, \ldots, I-1)$

$$
\frac{\varphi_{i}^{n}-\varphi_{i}^{n-1}}{\delta t}+(|u|+u) \frac{\varphi_{i+1}^{n}-\varphi_{i}^{n}}{2 \delta x}+(|u|-u) \frac{\varphi_{i-1}^{n}-\varphi_{i}^{n}}{2 \delta x}
$$




$$
=d \frac{\varphi_{i+1}^{n}-2 \varphi_{i}^{n}+\varphi_{i-1}^{n}}{(\delta x)^{2}}-\mu \varphi_{i}^{n},
$$

where $\delta t$ is the time step and $\delta x$ the space step. The superscript $n$ corresponds to the time step. To simplify the notations, in what follows we will omit $n$, that is, we will write $\varphi_{i}$ instead of $\varphi_{i}^{n}$, and will keep the notation $\varphi_{i}^{n-1}$. Let us write equation (3.14) in the form

$$
a_{i} \varphi_{i-1}-c_{i} \varphi_{i}+b_{i} \varphi_{i+1}=-f_{i}, \quad i=2, \ldots, I-1,
$$

where

$$
\begin{gathered}
a_{i}=\frac{|u|-u}{2 \delta x}+\frac{d}{(\delta x)^{2}}, \quad b_{i}=\frac{|u|+u}{2 \delta x}+\frac{d}{(\delta x)^{2}}, \\
c_{i}=\frac{1}{\delta t}+\frac{|u|}{\delta x}+\frac{2 d}{(\delta x)^{2}}+\mu, \quad f_{i}=\frac{\varphi_{i}^{n-1}}{\delta t} .
\end{gathered}
$$

Consider now the approximation of the boundary conditions. If the ends of the branch correspond to the root $(x=0)$ or to the growing part (Figure $4 \mathrm{a})$, then the approximation of the boundary conditions here yields the expressions:

$$
-c_{1} \varphi_{1}+b_{1} \varphi_{2}=-f_{1}
$$

and

$$
a_{I} \varphi_{I-1}-c_{I} \varphi_{I}=-f_{I} .
$$

Specific expressions for $a_{1}, b_{1}, \ldots, f_{I}$ in the case of some particular boundary conditions can be given in a similar way.

In the case of the growth of a single branch, the numerical scheme is completely described by system (3.15), (3.17), and (3.18). The matrix of this system is tri-diagonal:

$$
\left(\begin{array}{ccccccccc}
-c_{1} & b_{1} & 0 & 0 & 0 & 0 & 0 & \ldots & 0 \\
a_{2} & -c_{2} & b_{2} & 0 & 0 & 0 & 0 & \ldots & 0 \\
\ldots & & & & & & & & \\
0 & \ldots & 0 & a_{i} & -c_{i} & b_{i} & 0 & \ldots & 0 \\
\ldots & & & & & & & & \\
0 & \ldots & 0 & 0 & 0 & 0 & a_{I-1} & -c_{I-1} & b_{I-1} \\
0 & \ldots & 0 & 0 & 0 & 0 & 0 & a_{I} & -c_{I}
\end{array}\right) .
$$

In order to solve a linear system with a tri-diagonal matrix (3.19), we can use the Thomas algorithm.

The situation is more difficult if there are several branches. Moreover, the tree can have an arbitrary structure (see, e.g., Figure 3b). There are nodes from which several branches end or begin. Such nodes will also be called boundary nodes. Each of them will be numbered, and the value of $\varphi$ at the $k$-th node is denoted by $\varphi_{k}$. The boundary conditions in the 
conventional sense are not imposed in such nodes. Instead, we should take into account conservation of species and fluxes.

Let there exist $J$ branches leaving the boundary node $k$. Then $\varphi_{j, 1}=\varphi_{k}$ at $j=1, \ldots, J$. The values of $\varphi$ at the second node of each branch will be denoted by $\varphi_{j, 2}$, at the third $\varphi_{j, 3}$, and so on. Then the conservation of $\varphi$ at the node $k$ can be written as follows:

$$
\sum_{j=1}^{J}\left(d \frac{\varphi_{j, 2}-\varphi_{k}}{\delta x}-\mu \frac{\delta x}{2} \varphi_{k}-\frac{\delta x\left(\varphi_{k}-\varphi_{k}^{n-1}\right)}{2 \delta t}-\frac{\left|u_{j}\right|-u_{j}}{2}\left(\varphi_{k}-\varphi_{j, 2}\right)\right)=0 .
$$

If there are $J$ branches entering the boundary node $k$, then the finite difference scheme at this node will be:

$$
\sum_{j=1}^{J}\left(d \frac{\varphi_{j, I-1}-\varphi_{k}}{\delta x}-\mu \frac{\delta x}{2} \varphi_{k}-\frac{\delta x\left(\varphi_{k}-\varphi_{k}^{n-1}\right)}{2 \delta t}-\frac{\left|u_{j}\right|+u_{j}}{2}\left(\varphi_{k}-\varphi_{j, I-1}\right)\right)=0 .
$$

If for some of the branches the numeration begins at the node $k$ and for some other it ends there, then the finite difference scheme can be represented as a superposition of (3.20) and (3.21).

To summarize, we recall that, in the internal nodes of the mesh, we use a three-point scheme (3.15). In the boundary nodes, we have expressions (3.20) or (3.21) (see Figure 4b) that include $\varphi_{k}$ and the values $\varphi_{j, I-1}$ or $\varphi_{j, 2}$ taken at the nodes nearest to the boundary node from the branches connected to this node. Thus we have a completely implicit approximation of our 1D problem.

We describe here the algorithm for the case in which the domain of the computation has several branches arbitrarily connected between each other. Moreover, the number of branches can change during the computation. form:

The finite difference scheme for the internal nodes of the $j$-th subsystem has the following

$$
\left\{\begin{array}{l}
-c_{1} \varphi_{k_{1}}+b_{1} \varphi_{2}=-f_{1}+\text { other terms } \\
a_{i} \varphi_{i-1}-c_{i} \varphi_{i}+b_{i} \varphi_{i+1}=-f_{i}, \quad i=2, \ldots, I-1 \\
a_{I} \varphi_{k_{2}}-c_{I} \varphi_{I-1}=-f_{I}+\text { other terms. }
\end{array}\right.
$$

The first and the last equations in system (3.22) are obtained from expressions (3.20) and (3.21) corresponding to $k_{1}$-th and $k_{2}$-th boundary nodes. The terms corresponding to the $j$-th branch are written explicitly; for other branches they are included in "other terms".

Instead of the tri-diagonal matrix (3.19) we have here an "almost tri-diagonal" matrix 
for the $j$-th branch:

$$
\left(\begin{array}{cccccccccc}
-c_{1} & b_{1} & 0 & 0 & 0 & 0 & 0 & \ldots & 0 & \text { other terms } \\
a_{2} & -c_{2} & b_{2} & 0 & 0 & 0 & 0 & \ldots & 0 & \\
\ldots & & & & & & & & & \\
0 & \ldots & 0 & a_{i} & -c_{i} & b_{i} & 0 & \ldots & 0 & \\
\ldots & & & & & & & & & \\
0 & \ldots & 0 & 0 & 0 & 0 & a_{I-1} & -c_{I-1} & b_{I-1} & \\
0 & \ldots & 0 & 0 & 0 & 0 & 0 & a_{I} & -c_{I} & \text { other terms }
\end{array}\right)
$$

Step 1. Transformation of matrix (3.23) to an "almost two-diagonal" form. Let us write the corresponding system (3.22) in the form

$$
\left\{\begin{array}{l}
-c_{1} \varphi_{k_{1}}+b_{1} \varphi_{2}=-f_{1}+\text { other terms } \\
\beta_{i} \varphi_{k_{1}}-\varphi_{i}+\alpha_{i} \varphi_{i+1}=-\gamma_{i}, \quad i=2, \ldots, I-1 \\
a_{I} \varphi_{k_{2}}-c_{I} \varphi_{I-1}=-f_{I}+\text { other terms }
\end{array}\right.
$$

where

$$
\begin{aligned}
\alpha_{i} & =\frac{b_{i}}{c_{i}-\alpha_{i-1} a_{i}}, \quad \beta_{i}=\frac{a_{i} \beta_{i}}{c_{i}-\alpha_{i-1} a_{i}} \\
\gamma_{i}=\frac{a_{i} \gamma_{i-1}+f_{i}}{c_{i}-\alpha_{i-1} a_{i}}, & i=3, \ldots, I-1
\end{aligned}
$$

and

$$
\alpha_{2}=\frac{b_{2}}{c_{2}}, \quad \beta_{2}=\frac{a_{2}}{c_{2}}, \quad \gamma_{2}=\frac{f_{2}}{c_{2}} .
$$

Thus, matrix (3.23) is reduced to the following one:

$$
\left(\begin{array}{cccccccccc}
-c_{1} & b_{1} & 0 & 0 & 0 & 0 & 0 & \ldots & 0 & \text { other terms } \\
\beta_{2} & -1 & \alpha_{2} & 0 & 0 & 0 & 0 & \ldots & 0 & \\
\ldots & & & & & & & & & \\
\beta_{i} & \ldots & 0 & 0 & -1 & \alpha_{i} & 0 & \ldots & 0 & \\
\ldots & & & & & & & & & \\
\beta_{I-1} & \ldots & 0 & 0 & 0 & 0 & 0 & -1 & \alpha_{I-1} & \\
0 & \ldots & 0 & 0 & 0 & 0 & 0 & a_{I} & -c_{I} & \text { other terms }
\end{array}\right) .
$$

Step 2. Transformation of the matrix of system (3.24) to an almost diagonal form. Let 
us write the internal part of system (3.24) in the form:

$$
\left\{\begin{array}{l}
-c_{1} \varphi_{k_{1}}+b_{1} \varphi_{2}=-f_{1}+\text { other terms } \\
p_{i} \varphi_{k_{1}}-\varphi_{i}+q_{i} \varphi_{k_{2}}=-s_{i}, \quad i=2, \ldots, I-1, \\
a_{I} \varphi_{k_{2}}-c_{I} \varphi_{I-1}=-f_{I}+\text { other terms }
\end{array}\right.
$$

where

$$
\begin{gathered}
p_{i}=\alpha_{i} p_{i+1}+\gamma_{i}, \quad q_{i}=\alpha_{i} q_{i+1}, \\
s_{i}=\alpha_{i} s_{i+1}+\beta_{i}, \quad i=I-3, \ldots, 2
\end{gathered}
$$

and

$$
p_{I-2}=\gamma_{I-2}, \quad q_{I-2}=\alpha_{I-2}, \quad s_{I-2}=\beta_{I-2} .
$$

Now matrix (3.27) is reduced to the following one:

$$
\left(\begin{array}{cccccccccc}
-c_{1} & b_{1} & 0 & 0 & 0 & 0 & 0 & \ldots & 0 & \text { other terms } \\
p_{2} & -1 & 0 & 0 & 0 & 0 & 0 & \ldots & q_{2} & \\
\ldots & & & & & & & & & \\
p_{i} & \ldots & 0 & 0 & -1 & 0 & 0 & \ldots & q_{i} & \\
\ldots & & & & & & & & & \\
p_{I-1} & \ldots & 0 & 0 & 0 & 0 & 0 & -1 & q_{I-1} & \\
0 & \ldots & 0 & 0 & 0 & 0 & 0 & a_{I} & -c_{I} & \text { other terms }
\end{array}\right) .
$$

Step 3. Determination of the new values of $\varphi$ at the boundary nodes. For any branch we substitute the second equation $(i=2)$ from (3.28) into the first one, and the $(I-1)$-th equation to the last one. We obtain

$$
\begin{gathered}
\left(b_{k_{1}} p_{2}-c_{k_{1}}\right) \varphi_{k_{1}}+b_{k_{1}} q_{2} \varphi_{k_{2}}=-b_{k_{1}} s_{2}-f_{k_{1}}+\text { other terms } \\
\left(a_{k_{2}}-c_{k_{2}} q_{I-1}\right) \varphi_{k_{2}}-c_{k_{2}} p_{I-1} \varphi_{k_{1}}=c_{k_{2}} s_{I-1}-f_{k_{2}}+\text { other terms }
\end{gathered}
$$

Let us consider the first and the last equations in (3.32). We see that only the boundary nodes enter these equations. "Other terms" now also contain only the corresponding boundary nodes. Therefore, the boundary nodes form a complete system which allows us to find the unknowns $\varphi$ in all boundary nodes.

Step 4. Determination of new values in the internal nodes. When the values of $\varphi$ are found in all boundary nodes, we can obtain new values of $\varphi$ in the internal nodes for all branches using the corresponding internal equations from system (3.28).

This algorithm is applicable for an arbitrary connection of subsystems. We will finish this section with a short discussion of the sensitivity of the results with respect to numerical 
discretization. Convergence of the numerical results when we decrease the time and the space steps occurs when we consider finite and not very big time intervals. The situation is much more complex if we consider longtime behavior. Figure 4 shows final (stationary) solutions for different space steps. The absence of convergence of the results can be related to structural instability specific for this model and important for growth of biological organisms (see the discussion in [3]).
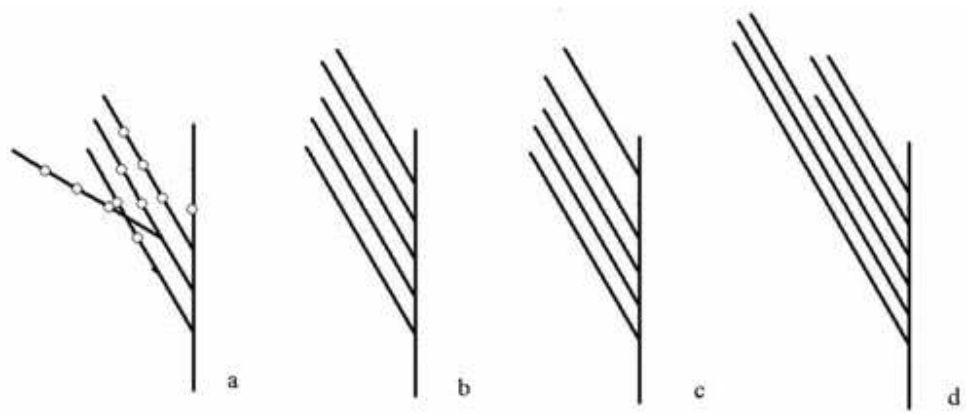

Figure 4: The final (stationary) form of the plant for different space steps: for each figure, the step is half of the previous one.

\section{Branching pattern}

A typical example of plant growth in the 1D model with branching is shown in Figure 5. In the beginning of the evolution there is a single branch which grows with an approximately constant speed until the growth period is finished. The apical meristem is located at the upper end of the interval. The plant hormones auxin and cytokinin are produced there and are transported along the whole branch. It can be diffusive or convective transport. Cytokinin can also be produced in the roots. This is taken into account through the boundary condition at $x=0$.

If at some point of the branch the concentrations of auxin and of cytokinin take on some prescribed values, then a new bud appears. In the simulation shown in Figure 5, there are five buds that appear one after another at an approximately equal distance. Each of the buds contains its own apical meristem with some value of the GM-factor $R$. When a new bud appears, the initial value $R_{0}$ is prescribed. It can be some given constant or it can depend on some factors (on plant hormones or on the value of $R$ in the apical meristem of the main growing branch). After that, the value of $R_{i}$ in the bud evolves according to the same equation (see (2.4)). 

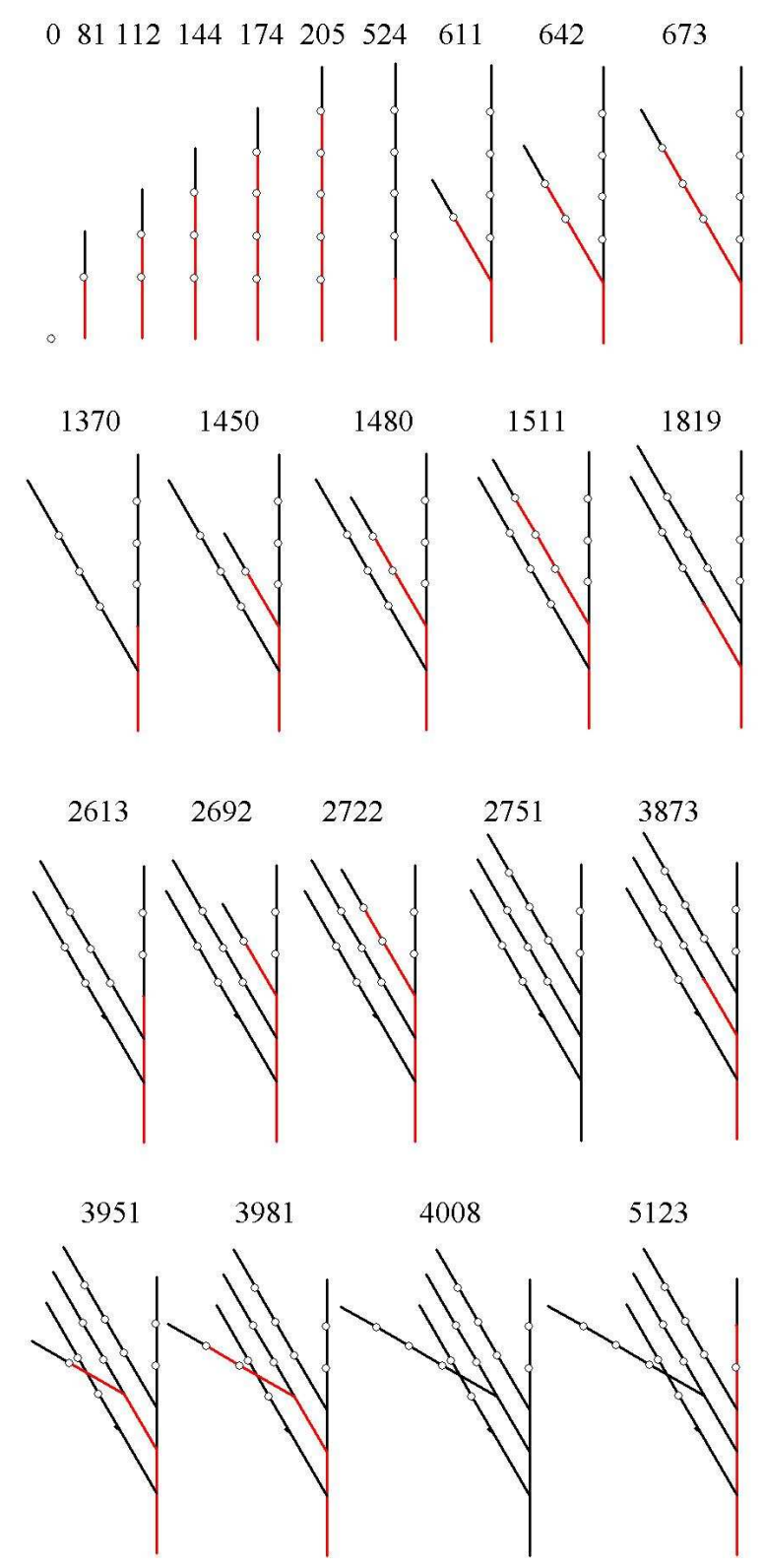

Figure 5: Time evolution of the plant. The figure shows the moments in time when new buds or branches appear. Convective flux of nutrients is shown in red. 
When $R_{i}$ becomes larger than $R_{f}$, proliferation begins and a new branch starts growing from the bud. As it is shown in [3], growth of branches can be stepwise, that is a branch has periods of growth and of rest during which the speed of its growth equals zero. When the main branch stops growing, under appropriate conditions a new branch can appear from another bud.

As it is discussed above, we consider diffusive and convective fluxes of nutrients. Diffusive flux acts throughout all branches, whereas convective flux is directed to growing branches. This is related to the continuity equation for the incompressible fluid. The routes of convective flux of nutrients is shown in red in Figure 5.

Thus, initiation of growth of new branches is determined by the interplay between the concentrations of nutrients and of the growth factor. In the example presented in Figure 5 there are five buds formed on the main branch. Three of them give branches of the second generation with three new buds on each of them. Branches of the second generation appear one after another when the main branch stops growing. It is interesting to note that one of the buds on the main branch gives a rudimentary branch which stops growing right after it appears. There are also some branches of the third generation.
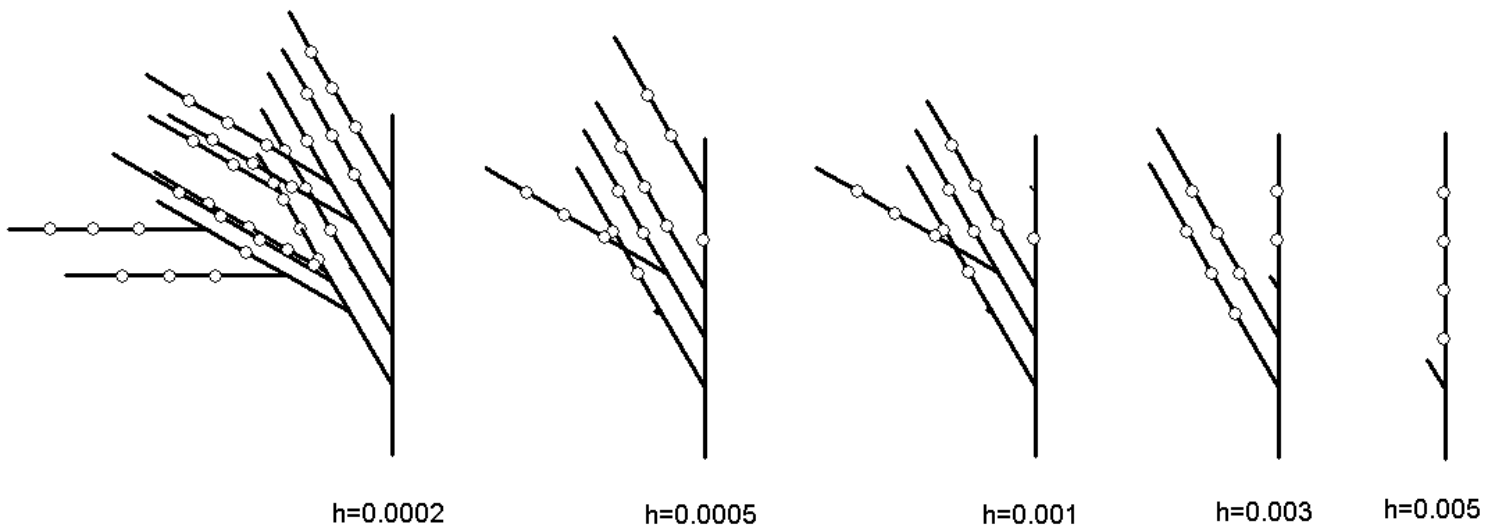

Figure 6: Final plant forms for different values of $h$.

Similar to the 1D case without branching, the final plant size decreases for larger $h$. In the case with branching, the final length is determined basically by the number of branches and not by their length (Figure 6). There are four generations of branches for $h=0.0002$, three of them for $h=0.0005$ and $h=0.001$, and only two generations for greater values of $h$. A possible explanation of the influence of $h$ on the final length from the point of view of nonlinear dynamics is given in [3].

Plant evolution in time can be influenced by the initial value of the GM-factor concentration in a new bud. We have described it in the case where $R_{0}=0.12$ (Figure 5). In fact, it is the same for all values of $R_{0}$ between 0.01 and 0.12 . Further increase of this parameter 
changes the plant evolution (Figure 7). When the first bud appears, it does not stay dormant but gives a new branch right away. It grows at the same time with the main branch. The difference between the two cases is determined by the behavior of solutions of equation (2.4). As we have already discussed, when a new bud appears, we prescribe it an initial value $R_{0}$ of the GM-factor concentration. We recall that the concentration of nutrients $C$ is a function of space and time. Its value $C\left(x_{0}, t_{0}\right)$ at the new bud determines the right-hand side of this equation: it equals zero at $R=0$ and at two positive values of $R$, it is negative between first two zeros and positive between the second two. If the initial value $R_{0}$ is at the interval of negativity, then the solution rapidly decreases, and the bud remains dormant. When the main branch stops growing, the concentration of nutrients increases. When its value $C\left(x_{0}, t\right)$ at the bud approaches 1, the right-hand side of equation (2.4) becomes positive for small positive values of $R$. The concentration of the GM-factor starts growing. After some time it can reach the critical value which is necessary for the new branch to grow. However, it may happen that another branch will start growing before this one. Then the concentration of nutrient can drop down again, and the concentration of the GM-factor may also decrease.
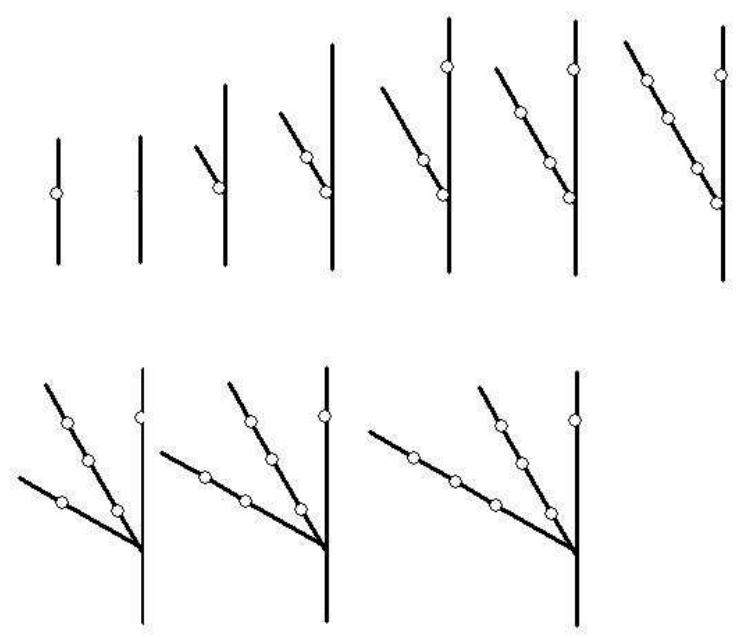

Figure 7: Evolution of the plant structure in time, $h=0.0005, R_{0}=0.13$. Increase of $R_{0}$ changes completely the plant structure.

Formation of new buds and growth of new branches is determined by a complex interaction of plant hormones, nutrients and mitotic factors. The plant structure depends on the values of the parameters. Figure 8 shows the simulations where the buds are double and give symmetric branches. The symmetry of the plant growth is prescribed by the algorithm. 
Consumption of nutrients and production of plant hormones are different in this case in comparison with the case without symmetry. Hence the number of new buds and the whole plant organization will also be different.

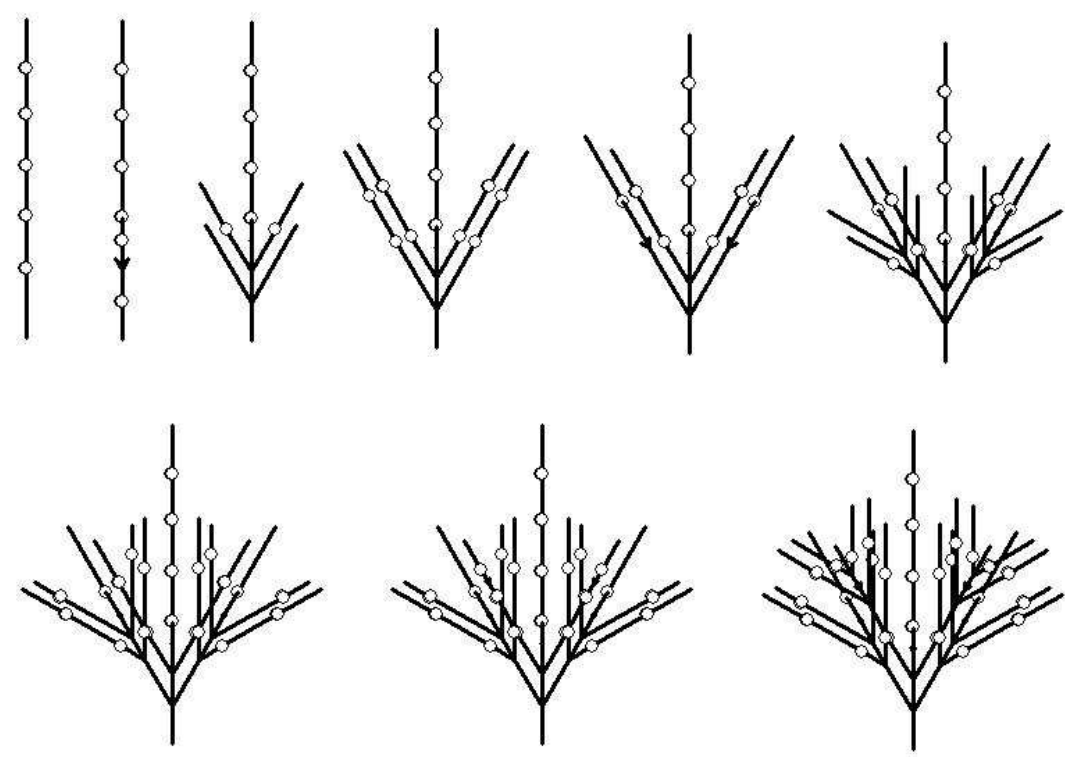

Figure 8: Plant evolution in the case of a double bud and $R_{0}=0.12$.

\section{References}

[1] Krug, H., Liebig, H.-P.: International Symposium on Models for Plant Growth, Environmental Control and Farm Management in Protected Cultivation. ISHS Acta Horticulturae, 248 (1989)

[2] Godin, C., et al.: 4th International Workshop on Functional-Structural Plant Models. Publication UMR AMAP (2004)

[3] Bessonov, N., Volpert, V.: Dynamic Models of Plant Growth. Publibook, Paris (2006)

[4] Bessonov, N., Morozova, N., Volpert, V.: Branching Pattern in Plants. Bull. Math. Biology. 70, no. 3, 868-893 (2008)

[5] Heisler, M.G., Ohno, C., Das, P., Sieber, P., Reddy, G.V., Long. J.A., Meyerowitz, E.M.: Patterns of Auxin Transport and Gene Expression during Primordium Development 
Revealed by Live Imaging of the Arabidopsis Inflorescence Meristem. Current Biology, 15, 1899-1911 (2005)

[6] Treml, B.S., Winderl, S., Radykewicz, R., Herz, M., Schweizer, G., Hutzler, P., Glawischnig, E., Ruiz, R.A.: The Gene ENHANCER OF PINOID Controls Cotyledon Development in the Arabidopsis Embryo. Development, 139, no. 18, 4063-4074 (2005)

[7] Reinhardt, D.: Regulation of Phyllotaxis. Int. J. Dev. Biol. 49, 539-546 (2005)

[8] Smith, R.S., Guyomarc'h, S., Mandel, T., Reinhardt, D., Kuhlemeier, C., Prusinkiewicz, P.: A Plausible Model of Phyllotaxis. PNAS, 103, no. 5, 1301-1306 (2006)

[9] Jonsson, H., Heisler, M.G., Shapiro, B.E., Meyerowitz, E.M., Mjolsness,E.: An AuxinDriven Polarized Transport Model for Phyllotaxis. PNAS, 103 no. 5, 1633-1638 (2006)

[10] Fleming, A.J.: Formation of Primordia and Phyllotaxy. Current Opinion in Plant Biology $8,53-58(2005)$

[11] Reinhardt, D., Mandel, T., Kuhlemeier, C.: Auxin Regulates the Initiation and Radial Position of Plant Lateral Organs.Plant Cell 12, 507-518 (2000)

[12] Reinhardt, D., Pesce, E.R., Stieger, P., Mandel, T., Baltensperger, K., Bennett, M., Traas, J., Friml, J., Kuhlemeier, C.: Regulation of Phyllotaxis by Polar Auxin Transport, Nature 462, 255-260 (2003)

[13] Blilou, I., Xu, J., Wildwater, M., Willemsen, V., Paponov, I., Friml, J., Heidstra, R., Aida, M., Palme, K., Scheres, B.: The PIN Auxin Efflux Facilitator Network Controls Growth and Patterning in Arabidopsis Roots, Nature, 433, 39-44 (2005)

[14] Vernoux, T., Kronenberger, J., Grandjean, O., Laufs, P., Traas, J.: PIN-FORMED 1 Regulates Cell Fate at the Periphery of the Shoot Apical Meristem. Development, 127, 5157-5165 (2000)

[15] Galweiler, L., Guan, C., Muller, A., Wisman, E., Mendgen, K., Yephremov, A., Palme, K.: Regulation of Polar Auxin Transport by AtPIN1 in Arabidopsis Vascular Tissue. Science, 282, 2226-2230 (1998)

[16] Aida, M., Vernoux, T., Furutani, M., Traas, J., Tasaka, M.: Roles of PIN-FORMED1 and MONOPTEROS in Pattern Formation of the Apical Region of the Arabidopsis Embryo. Development, 129, 3965-3974 (2002)

[17] Stieger, P.A., Reinhardt, D., Kuhlemeier, C.: The Auxin Influx Carrier is Essential for Correct Leaf Positioning. Plant J. 32, 509-517 (2002) 